\title{
Identifying the mitochondrial DNA mutations that cause cancer
}

Mutations that affect the
mitochondrial DNA may play a
key role in cancers. However,
the exact mutations that are
involved in cancer are still
unknown. Dr Fatimata Mbaye
and collaborators, from the
University of Dakar in Senegal
examined the sequences of two
regions of the mitochondrial
genome to identify and
compare the mutations
occurring in different cancers.
Understanding the relationship
between mitochondrial
mutations and their roles in
cancer could eventually lead to
mitochondrial DNA being used
as an oncological biomarker to
diagnose cancer at earlier stages.

espite cancer being one of worldwide, detecting cancers early enough to treat them is still a challenge today. Cancer is complex. Various mechanisms can be involved and some of them have yet to be understood.

Most cancers are characterised by genetic instabilities. Somatic mutations (i.e. alterations in DNA that occur after conception) accumulate and transform the cells, for example giving them properties. White most of the gene is contained in the ot of the genome genome is contained in mitochondria, and there is growing evidence that somatic mitochondrial mutations colld be linked to cancer.

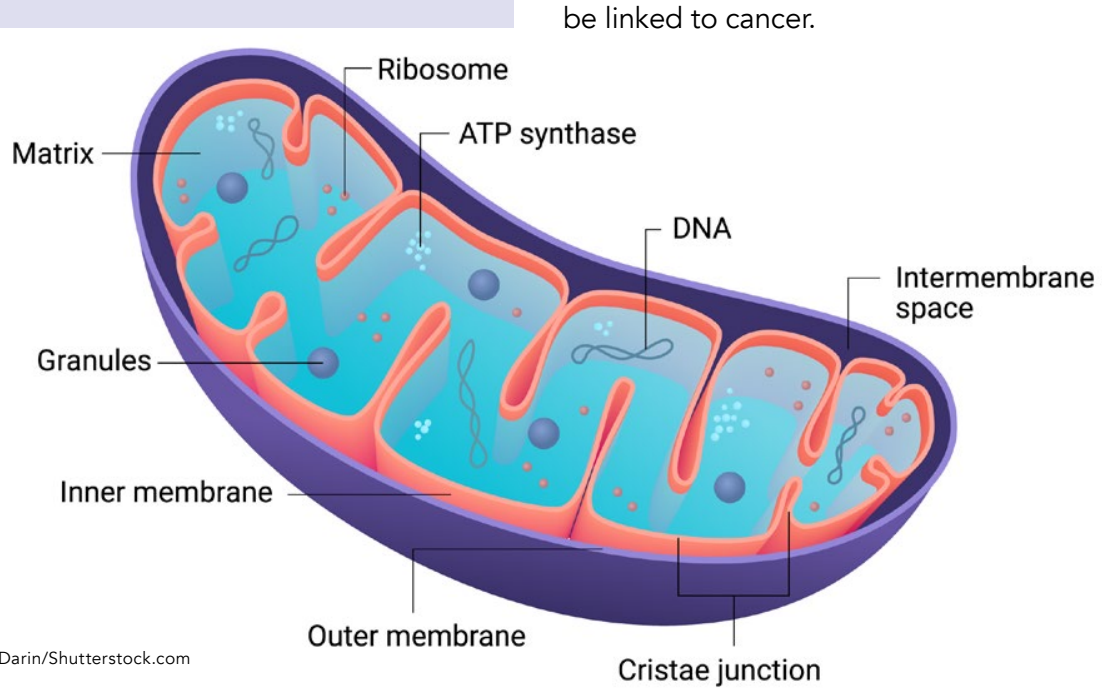

THE POWERHOUSE OF THE CELL Mitochondria are organelles that are known as the powerhouses of the cell. energy needed to power all the biochemical reactions that happen in the cell. This chemical energy is stored in small molecules called adenosine triphosphate (ATP).

When a source of energy such as carbohydrates (sugars and fibres), fats or proteins enters the cell, it is brought into pritochondria and converted hrough a cyccession of reacions called the Krebs of the Krebs cycle are then used in second metabolic pathway named oxidative phosphorylation. It is through oxidative phosphoylation that ATP, the universal energy currency in cells, is generated.

Reactive oxygen species (ROS) are side products of oxidative phosphorylation. ROS play diverse roles that are beneficial to the body. They are essential in the cardiovascular and (n) ROS can also be toxic: they possess an unpaired electron which makes them highly reactive and thus able to damage Proteins, lipids and nucleic acids (such body therefore requires a bane its ROS levels as an excess will lead to

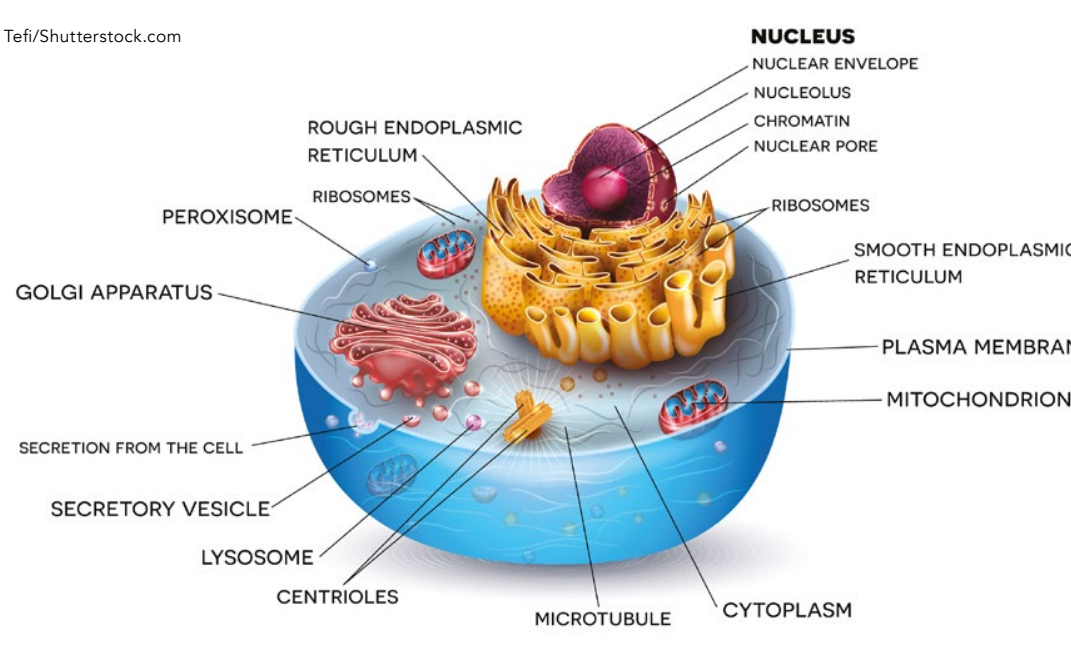

\section{eventually lead to
at earlier stages.}

oxidative stress while a level that is too low will lead to reductive stresses tha can cause pathologies ranging

\section{MITOCHONDRIAL DNA}

Beside their characteristic role as the

powe another specificity: they are the only place outside the nucleus where DNA can be found. While the nuclear genome is linear, mitochondrial DNA is a small circular chromosome. Each cell

Finding oncological biomarkers could help diagnosing oral cancers at an earlier stage.

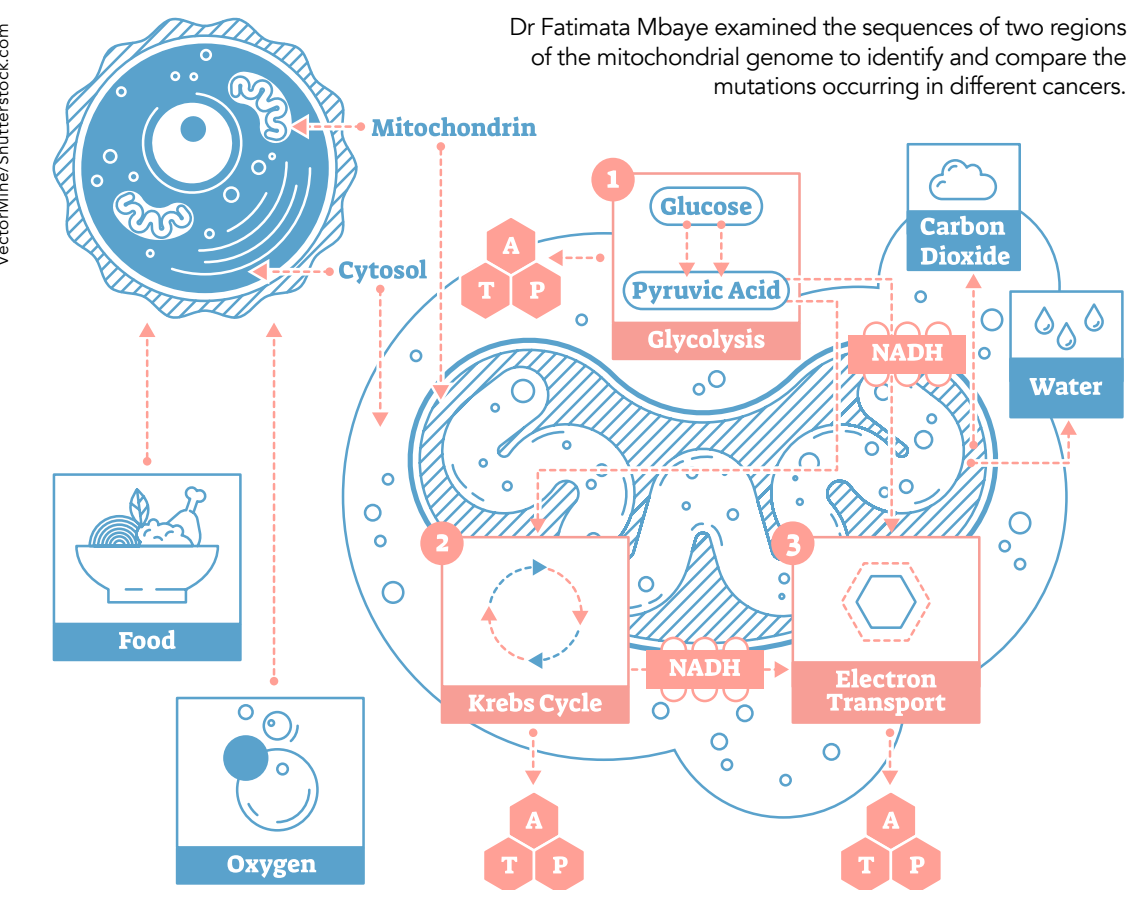

by the mitochondrial genome all take pathway that entive phosphonlation generate energy.

\section{FREQUENT MITOCHONDRIAL} MUTATIONS

the naturally in every cell. However, the rate of mitochondrial DNA mutation is several times higher than the ate for nuclear DNA. A first explanation is that, in the nucleus, a system called mismatch repair recognises and repairs can therefore be reverted. In the

mitochondria, this reparation system is less efficient. A second explanation is that ROS, which are produced during the oxidative phosphorlation hat takes Dlace in the mitochondia, cand

Mitochondrial mutations may modify the function of normal oxidative phosphorylation chain and lead to mproper production of genotoxic reactive oxygen species. This Scientists widely accept the idea that mitochondria play an important role in the ageing processes of both cells and individuals. Increased accumulation of mitochondrial DNA mutations has been reported in ageing tissues such as brain, skeletal muscle, and fibroblasts and in many pathological conditions including neurologic, metabolic, and

Mitochondrial mutations have also been frequently observed in human cancers and proposed as important exact mitochondrial mutations that are responsible for the pathogenesis of cancer remain unclear. To shed light on this, Dr Mbaye and her team examine mitochondrial mutations in cancer and focus on two specific regions: the D-Loop region and the MT-CYB gene.

THE D-LOOP REGION Dr Mbaye and her collaborators focus on the D-Loop region of mitochondria DNA because this region is crucial for replication and expression of the transcriptiol genome. It contains essential for gene expression. It also contains the leading-strand origin of 


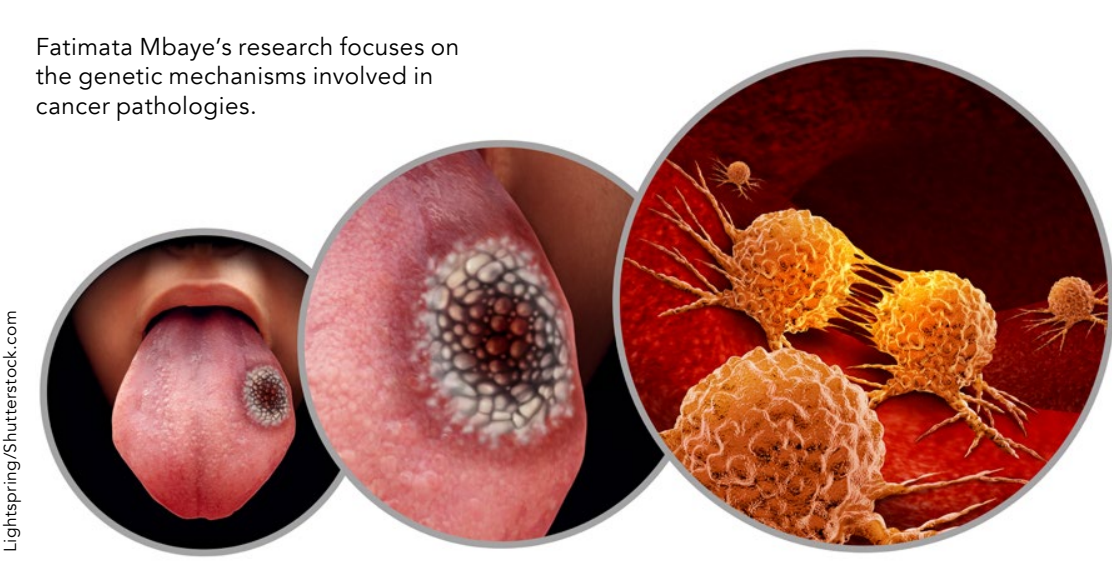

replication, a third strand of DNA that initiates replication of the genome. This third strand gives this region its distinctive triple-stranded DNA struc
that makes it more susceptible to

somatic mutations.

THE MT-CYB GENE

Dr Mbaye and collaborators' research

has also honed in on the MT-CYB gene,

which provides
instructions for

instructions for

called Cytochrome

B. Cytochrome

$B$ is one of 11

components of

the MT-CYB gene: almost $80 \%$ of the mutations that were observed were in the D-Loop region while only $20 \%$ affected the MT-CYB gene. This does not necessarily mean that mutations naturally occur more frequently in the D-Loop region than in the MTCYB gene, but suggests that mutations in severe consequences and favour cancer be attributed to a specific cannot For example, it is unclear which of the two mitochondrial regions (MT CYB or the D-Loop) is associated with tumorigenesis, including changes in the cell cycle, cell death, and differentiation. It is also unknown which of the mutations in the two regions are responsible for giving cancer its invasive properties.

\section{SIMILARITIES IN}

DIFFERENT CANCERS

Now that Dr Mbaye and her team

provided evidence for the theory that

most oral cancers contain mitochondri mutations, the next step will be to

examine mutations in other cancers.

(n) that affects $20.7 \%$ of women. Ovarian

Among the oral cavity tumour tissues, almost $90 \%$ carried mitochondrial mutations. the MT-CYB
than mutations occurring in other parts of the mitochondrial genome.
Dr Mbaye also observed a high
heterogeneity within tumours: not heterogeneity within tumours: not all cells within a given tumour carry the same mutations. This heterogeneity

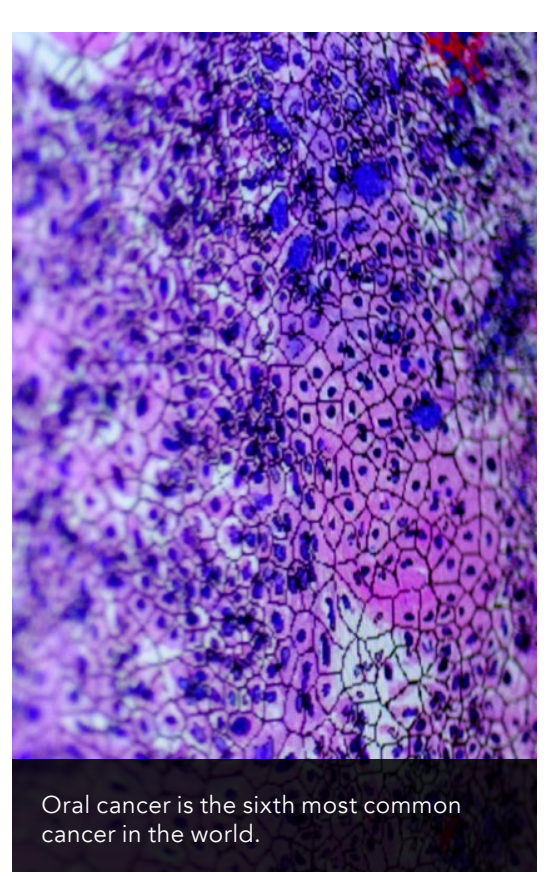 sequences of cancer, ovarian cancer and oral cancer samples. Their aim will be to compare the mutation profiles and identify the mitochondrial mutations that are shared in the three different cancers. Finding similarties between mutation profiles pould suggest a common pathologic
TOWARDS NEW
ONCOLOGICAL BIOMARKERS?
mutations and the well-established
mitochondrial dysfunction in cancer, the mitochondrial genome is, according to Dr Mbaye and her team, an under- explored avenue for insight into cancer pathogenesis. Their study confirms that a high rate of mitochondrial mutation is visible in cancer. Further studies are now required to elucidate the
relationship between the mitochondrial mutations, the pathological process and the survival rate in cancer patients, by understanding which mutations play a key role in cancer, mitochondrial Dource for be an incersting candidate source for biomarkers that would help
detect cancers at an earlier stage.

, which is involved in the oxidative

phosphorylation pathway. The MT-CYB

highly variable both amocause it is

among human individuals.

MITOCHONDRIAL MUTATIONS

\section{IN ORAL CANCER}

NORAL CANCER

cancer in the world. Most oral cancers

are detected at an advanced stage,

which is not ideal for patient care

and treatment. Finding oncological

biomarkers could help to diagnose

oral cancers at an earlier stage. With

this in mind, Dr Mbaye examined

the MT-CYB and D-Loop sequences

of the mitochondrial genome in 45

oral cavity cancers: the D-Loop and

DNA derived from control and tumo

tissues were analysed, and any DNA

sequence differences were regarded as

somatic mutations.

Among the oral cavity tumour tissues, almost $90 \%$ carried mitochondrial

mutations. Mutations were much more
frequent in the D-Loop region than in

\section{Behind the Research}

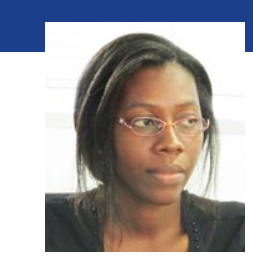

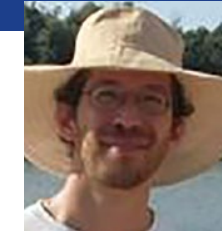

Dr Jean-

Baptiste Lamy

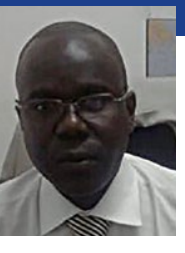

Professor

Mbacké

Sembene

Research Objectives

Fatimata Mbaye and her collaborators' research explores the genetic mechanisms involved in cancer pathologies.

\section{Detail}

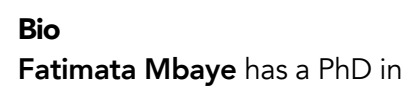
activities focus on the genetic

pathologies. She is MCA at Cheikh Anta Diop University in Dakar,
courses in bioinformatics. Population Genetics. Her research pathologies. She is MCA at Cheikh Anta

\section{Jean-Baptiste Lamy (PharmD, PhD) is a senior lecturer at University Paris 13 in the LIMICS laboratory. He teaches bioinformatics and medical informatics. His main research interests are information and knowledge visualisation, knowledge clinical decision support. He designed innovative visual approaches such as the patented VCM medical iconic language,}

\section{References}

Toure S., Mbaye F., Gueye M.D., Fall M., Dem A., Lamy J.B., \& Sembene M. (2019). Somatic Mitochondrial. Mutations in Oral Cavity Cancers among Senegalese Patients. Asian Pacific Journal of Cancer Prevention: APJCP, 20(7), 2203-2208. https://doi.org/10.31557/ APJCP.2019.20.7.2203

LIMLS \&

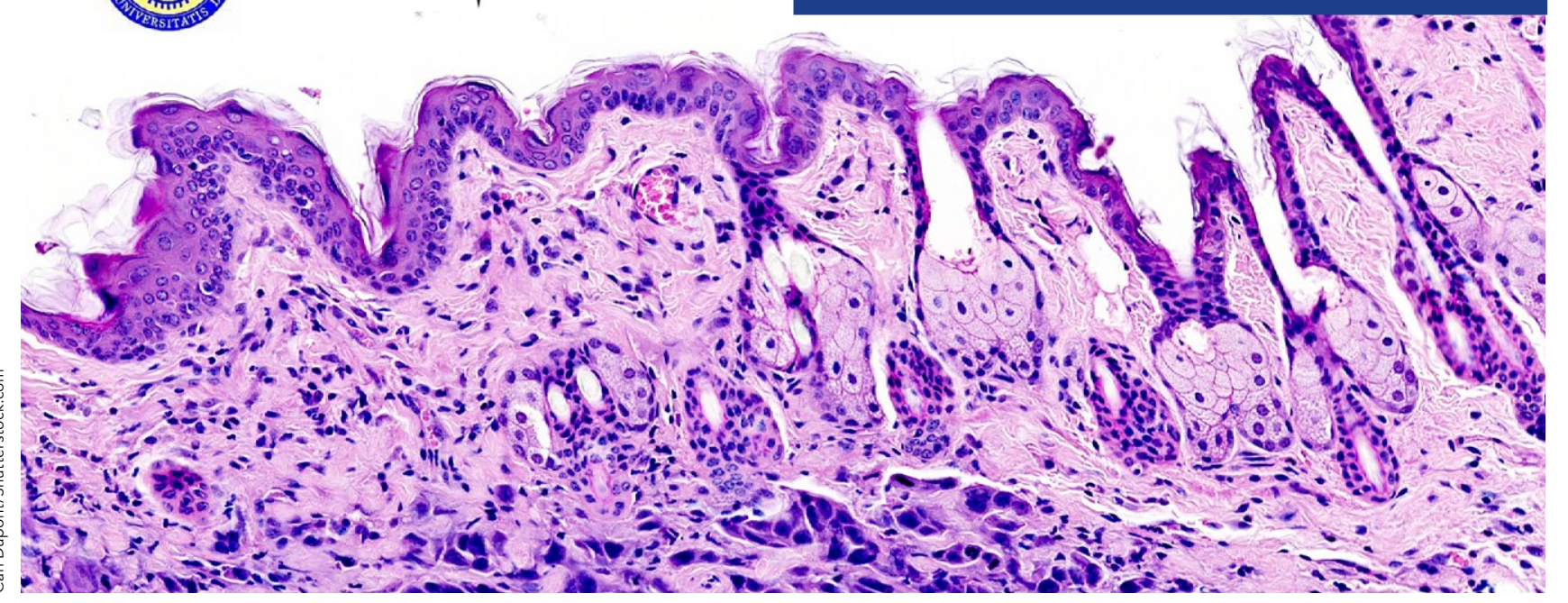

